\title{
Primary Healthcare Response to COVID 19 in a District of Callao, Peru
}

\author{
Juan Morales*, ${ }^{*}$, Marlene Raquel Basilio-Rojas², Maria Rosa Gonzales-Gonzales², Ana Paula Goyzueta ${ }^{3}$ \\ ${ }^{1}$ E-Health Research Center, University of Sciences and Humanities, 15306, Peru
}

${ }^{2}$ Mi Peru Health Center, DIRESA Callao, 07056, Peru

${ }^{3}$ Faculty of Health Sciences, University of Sciences and Humanities, 15306, Peru

\begin{tabular}{l} 
A R T I C L E I N F O \\
\hline Article history: \\
Received: 01 August, 20 \\
Accepted: 19 September, \\
Online: 12 October, 202 \\
\hline Keywords: \\
Primary Health Care \\
Home Care Services \\
Coronavirus Infections \\
Peru
\end{tabular}

Peru

\begin{abstract}
A B S T R A C T
Objectives: Describe the clinical characteristics and home care of patients diagnosed with COVID-19 in a Primary Healthcare Facility of the "Mi Peru" district, in the Callao Region, Peru. Materials and methods: Observational and descriptive cross-sectional study. A total of 84 subjects with positive results for Rapid Test IgM / IgG or molecular test (RTPCR) participated. Results: Of the sample, 59.5\% $(n=50)$ were males, $60.7 \%(n=51)$ aged 30 to 59 years and $21.4 \%(n=18)$ aged 60 to over of age, $67.9 \%(n=57)$ were positive on the rapid test and the rest on the molecular test. Diabetes and high blood pressure were the main pathological antecedents. Among the most frequent clinical manifestations were general malaise, fever and cough, with 40.5\% $(n=34), 36.9 \%(n=31)$ and $31 \%(n=26)$, respectively. The most frequently prescribed medications were acetaminophen and azithromycin, 25\% $(n=21)$, and $21.4 \%(n=18)$, respectively. Regarding home monitoring of patients diagnosed with COVID-19, the first week was performed with a higher proportion of those affected, progressively decreasing in the second week. Conclusions: Evidence of an important response to COVID-19 is shown in the scenario of the first level of care. Mild and moderate cases can be treated outpatient and complemented with home monitoring, contributing to the containment of the pandemic.
\end{abstract}

\section{Introduction}

COVID-19 is a disease caused by type 2 coronavirus causing severe acute respiratory syndrome (SARS-CoV-2), originated in Wuhan (China) in December 2019, and on 30 January 2020, the World Health Organization (WHO) has declared it as a public health emergency of international importance [1]. Globally, the number of positive cases and the number of deaths from COVID19 are terrifying, where the region of Americas recorded the highest number of cases in the world, led by the United States of North America and Brazil [2]. In Peru, the first case of COVID19 was confirmed on $6^{\text {th }}$ of March 2020 [3], since then the number of cases and deaths have had a sustained increase, Lima and Callao manifested the highest percentage [4].

In China, the majority of COVID-19 cases were symptomatic, the proportion of severe cases and the case fatality rate were $25.6 \%$ (17.4 to 34.9 ) and $3.6 \%$ (1.1 to 7.2$)$, respectively; older patients and those with comorbidities resulted in more severe symptoms

${ }^{*}$ Corresponding author: Juan Morales, E-Health Research Center, University of Sciences and Humanities. Lima, Peru. Email: mdjuanmorales@gmail.com www.astesj.com

https://dx.doi.org/10.25046/aj0505105
[5]. People with high blood pressure, diabetes, chronic obstructive pulmonary disease, cardiovascular and cerebrovascular disease were associated with serious complications and COVID-19 fatality [6]. Of the patients hospitalized for COVID-19, 20\% required the use of Intensive Care Units (ICU) and 13.9\% (95\% CI 6.2-21.5\%) of hospitalized patients had fatal results [7].

COVID-19 pandemic in addition to the enormous consequences for health systems. It also has an impact on the economic plane and on the labor market, both in supply and demand [8]. Social distancing, self-isolation, and travel restrictions forced a decrease in the workforce in all economic sectors and caused the loss of many jobs[9].

For COVID-19, to date, there are no specific medications or vaccines available [10]. So hospital support therapy remains the top priority; however, primary healthcare can play an important role in the COVID-19 response by differentiating patients with respiratory symptoms from those with COVID-19, making an early diagnosis, helping vulnerable people cope with their anxiety 
towards the virus, and reducing the demand for hospital services [11].

In the fight against COVID-19, hospital establishments are the main protagonists; however, the growing demand for services has exceeded the response capacity of hospitals. For this reason, the establishments of the first level of care, which are generally more precarious, assume a more active role and act in two settings, intramural and extramural. The objective of the present study was to describe the clinical characteristics and home care of patients diagnosed with COVID-19 in a Primary Healthcare Facility of the "Mi Peru" district, in the Callao Region, Peru.

\section{Materials and Methods}

\subsection{Design}

An observational and descriptive cross-sectional study was held at "Mi Peru" Primary Healthcare Facility, located in the district of the same name, which belongs to the Callao Region. The source of information was the emergency medical records and monitoring records with a positive result for COVID-19. The attentions made by the special team COVID-19, in the period of April and May 2020, were considered.

\subsection{Population and sample}

Our study population consisted of all patients with a positive test report for COVID-19. All subjects who had a positive result for COVID-19, relevant sociodemographic, and clinical data, as well as information from the home follow-up, were included.

Of a total of 91 cases enrolled in the COVID-19 positive patient registry, seven were excluded because they did not have a record of confirmatory laboratory results. The sample was made up of 84 confirmed cases.

\subsection{Study variables and instruments}

COVID-19: Disease caused by the SARS-CoV-2 virus (Severe acute respiratory syndrome coronavirus 2 ), the diagnosis of which was established using the Rapid IgM / IgG Test or molecular test (RT-PCR) for COVID-19, according to the national technical document [12].

Comparison variables: Sex, age, type of test for the diagnosis of COVID-19, the establishment of diagnosis, contacts with people suspected of COVID-19, hospitalization, clinical condition, clinical manifestations, vital functions, body weight, saturation of oxygen, medical prescription, pathological history, and home care services of confirmed cases.

\subsection{Statistic analysis}

Data were obtained from the emergency medical records and from the home care services registry, then entered into a matrix in the IBM SPSS Statistics 23 program. Qualitative variables were distributed according to frequencies and represented in tables and graphs. For the variables belonging to the vital functions, the means and extreme values were determined.

\subsection{Ethical considerations}

Access to information sources was made with the authorization of the Chief Medical Officer of the "Mi Peru" Health Center and precautions were taken to safeguard the confidentiality of the data as indicated in the Declaration of Helsinki of the World Medical Association [13]. The Ethics Committee of the University of Sciences and Humanities (CEI Act No. 017-2020) approved the research project.

Table 1: Epidemiological characteristics of people diagnosed with COVID-19, District of "Mi Peru", Callao Region, 2020.

\begin{tabular}{|c|c|c|}
\hline Epidemiological characteristics & $\mathrm{n}$ & $\%$ \\
\hline Total & 84 & 100 \\
\hline \multicolumn{3}{|l|}{ Sex } \\
\hline Female & 34 & 40.5 \\
\hline Male & 50 & 59.5 \\
\hline \multicolumn{3}{|l|}{ Age (years) } \\
\hline $12-17$ & 2 & 2.4 \\
\hline $18-29$ & 13 & 15.5 \\
\hline $30-59$ & 51 & 60.7 \\
\hline$\geq 60$ & 18 & 21.4 \\
\hline \multicolumn{3}{|l|}{ Type of test } \\
\hline Molecular & 27 & 32.1 \\
\hline Rapid & 57 & 67.9 \\
\hline \multicolumn{3}{|l|}{ Health facility } \\
\hline Local Health Center & 78 & 92.9 \\
\hline Derivated from a hospital & 6 & 7.1 \\
\hline \multicolumn{3}{|l|}{ Contacts } \\
\hline No apparent contact & 65 & 77.4 \\
\hline Positive contact & 2 & 2.4 \\
\hline Contact with suspect & 2 & 2.4 \\
\hline Sick family member & 11 & 13.1 \\
\hline Deceased relative & 3 & 3.6 \\
\hline Healthcare worker & 1 & 1.2 \\
\hline \multicolumn{3}{|l|}{ Hospitalization } \\
\hline No & 81 & 96.4 \\
\hline Yes & 3 & 3.6 \\
\hline \multicolumn{3}{|l|}{ Patient's condition } \\
\hline Favorable evolution & 78 & 92.9 \\
\hline Died & 6 & 7.1 \\
\hline Referrals to a hospital & 2 & 2.38 \\
\hline \multicolumn{3}{|l|}{ Medical history } \\
\hline Diabetes & 3 & 3.57 \\
\hline High blood pressure & 3 & 3.57 \\
\hline Tuberculosis & 1 & 1.19 \\
\hline Pregnant & 2 & 2.38 \\
\hline
\end{tabular}

\section{Results}

This study involved 84 patients of both sexes diagnosed with COVID 19, with a mean age of 45.3 years $(\mathrm{SD}=16.74$; Range: 15 to 98), with an average illness time of 8.65 days (Min. 3, Max. 21). 
Of the participants, $59.5 \%(\mathrm{n}=50)$ were male, $60.7 \%(\mathrm{n}=51)$ aged $30-59$ years and $21.4 \%(\mathrm{n}=18) 60$ or over. Of the sample, $67.9 \%(\mathrm{n}=57)$ were positive to the rapid test and the rest to the molecular test, $92.9 \%(n=78)$ were performed at the "Mi Peru" Health Center. Most of the participants had no contact with people suspected of COVID-19; however, $16.7 \%(\mathrm{n}=14)$ were in contact with a family member who was sick or died of COVID-19, 3.6\% $(n=3)$ were hospitalized, and $7.1 \%(n=6)$ of the sample died. Diabetes and high blood pressure were the main pathological antecedents among patients diagnosed with COVID-19 (Table 1).

Frequent clinical manifestations among patients diagnosed with COVID-19 were general malaise, fever and cough, with $40.5 \%(n=34), 36.9 \%(n=31)$ and $31 \%(n=26)$, respectively. Most frequently prescribed medications were acetaminophen and azithromycin, in $25 \%(\mathrm{n}=21)$ and $21.4 \%(\mathrm{n}=18)$, respectively (Figure 1). During the evaluation, the patients had vital functions outside the normal parameters (Table 2).

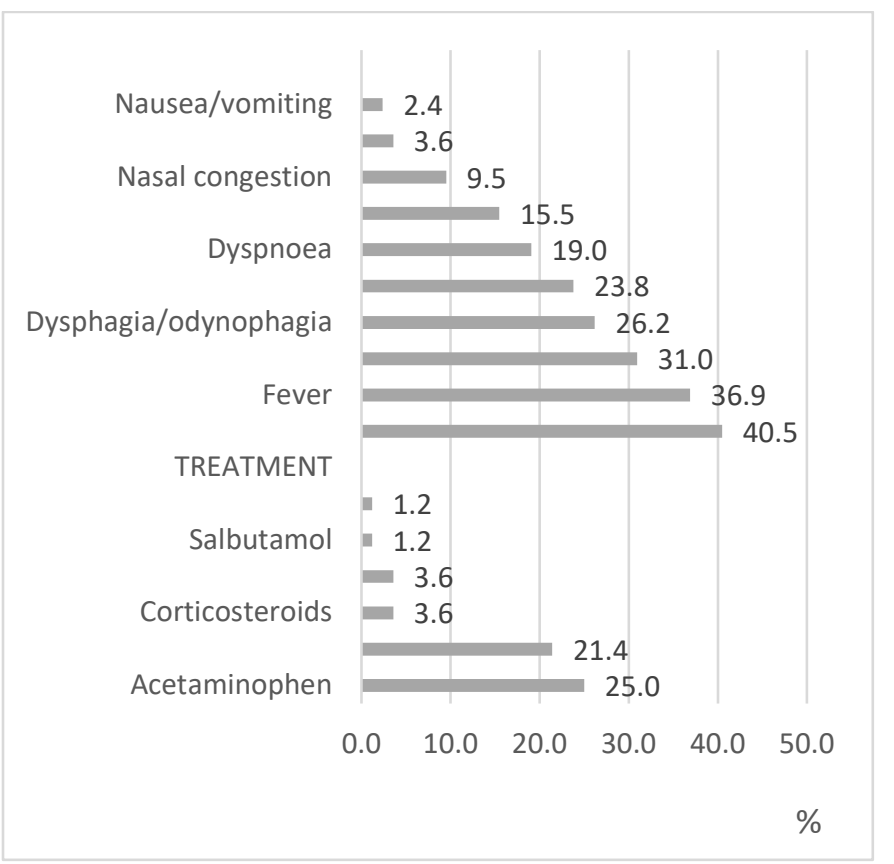

Figure 1: Clinical presentation and treatment in patients diagnosed with COVID19, "Mi Peru” District, Callao Region, 2020.

Table 2: Vital functions of patients affected by COVID-19, "Mi Peru" district, Callao Region, 2020.

\begin{tabular}{|lccc|}
\hline \multicolumn{1}{|c}{ Vital functions } & Mean & Min & Max \\
\hline Systolic blood pressure & 110.8 & 90 & 140 \\
Diastolic blood pressure & 67.2 & 40 & 80 \\
Heart rate & 91.15 & 65 & 116 \\
Breathing rate & 22.91 & 18 & 26 \\
Temperature & 37.15 & 36 & 39 \\
Oxygen saturation & 94.41 & 75 & 100 \\
Body weight & 76.77 & 54 & 103 \\
\hline
\end{tabular}

www.astesj.com
Regarding home health monitoring of patients diagnosed with COVID-19, the first week was performed with a higher proportion of those affected, progressively decreasing in the second week (Figure 2).

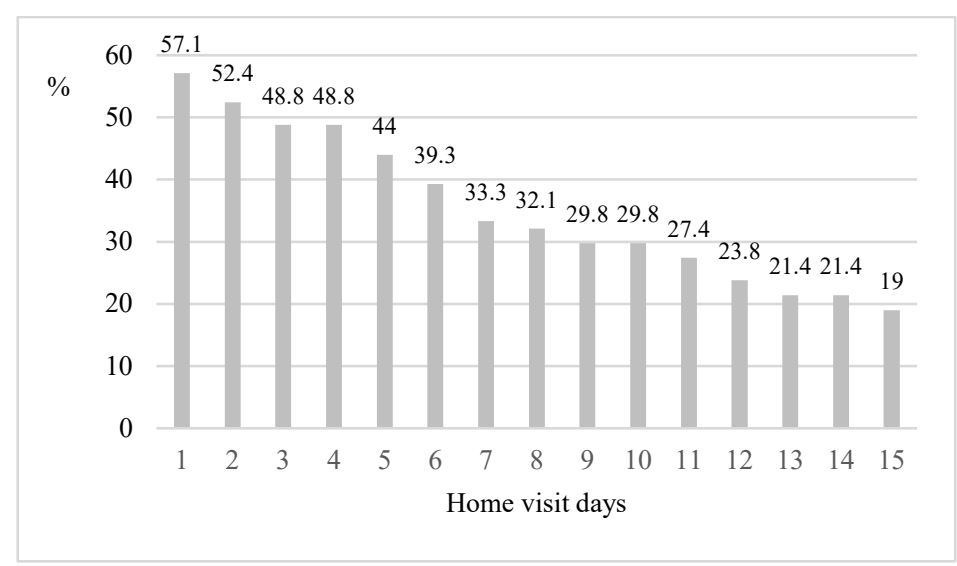

Figure 2: Home monitoring of patients diagnosed with COVID-19, "Mi Peru" district, Callao Region, 2020.

\section{Discussion}

In the present study, the majority of those affected by COVID19 were males, mainly adults (30-59 years) and older adults $(\geq 60$ years). Among the most frequent comorbidities in patients diagnosed with COVID-19 were diabetes mellitus and high blood pressure. Frequent clinical manifestations were general malaise, fever, and cough, with an illness time of approximately one week. Despite having vital functions outside normal parameters, most required only outpatient treatment and home monitoring.

Clinical presentation of COVID-19 is not yet fully clarified, the symptoms vary from mild to severe. To date, the most commonly reported symptoms are fever, cough, myalgia, dyspnea, and less common symptoms include headache, diarrhea, hemoptysis, and a runny nose. Mild symptoms recover after one week, while severe cases experience progressive respiratory distress that can lead to death; the average reported incubation duration was seven days and ranges from two to 14 days [14].

In our research, the most frequent comorbidity in patients diagnosed with COVID-19 was diabetes mellitus and high blood pressure, which are frequent problems in the country and primary healthcare establishments play an important role in control and treatment. These findings are consistent with studies conducted in China, which indicate that the most prevalent comorbidity was high blood pressure and diabetes. Patients with any comorbidity have poorer clinical outcomes, and a higher number of comorbidities is correlated with poorer clinical outcomes [15].

Based on the finding, two pregnant women had positive tests for COVID-19 and only received outpatient treatment. Symptoms of COVID-19 in pregnant women are not different from that of non-pregnant women; vertical transmission has not been reported either. However, they can cause fetal distress, miscarriage, respiratory distress, and premature delivery [16], they can also end in an emergency cesarean section for these complications [17].

Patients with mild symptoms are eligible for isolation, while patients with severe pneumonia require hospitalizations and frequent access to intensive care units [10]. Although to date there 
are no specific medications or vaccines available for the treatment of COVID-19, different drug therapies have been tried that have offered a limited level of evidence [10].

Regarding the drugs used in the health response, Azithromycin and Acetaminophen were the most frequently indicated drugs and are among the drugs included in the technical documents of the Ministry of Health (MINSA). According to the Technical Standard of the MINSA, for the management of moderate and severe cases in hospitalized patients, Chloroquine Phosphate or Hydroxychloroquine plus Azithromycin has been considered [12], after, at the proposal of the Peruvian Society of Hematology, Enoxaparin was incorporated in moderate and severe clinical scenarios [18]. In RM N ${ }^{\circ} 270-2020-M I N S A$, published on 8 May 2020, for mild cases, Hydroxychloroquine plus Ivermectin was considered; while for moderate to severe cases, Hydroxychloroquine, Azithromycin plus Ivermectin [19].

Although vital functions and oxygen saturation were found altered in the initial evaluation, only two patients needed reference for hospital management. In the Health Center of the "Mi Peru" district, the hospitalization service still does not work, so the figure of $3.6 \%$ of those hospitalized refers to patients who went alone to some other hospital in the Callao Region and were hospitalized.

Most of the patients were diagnosed at the "Mi Peru" Health Center, with an indication for outpatient treatment and monitored by phone and in person. In-person monitoring was carried out in their homes and consisted of clinical evaluation; verify treatment compliance, providing orientation, and comprehensive sanitary information. Extramural monitoring has been prioritized during the first week of evolution and responds mainly to insufficient human and technological resources. According to the Technical Standard of the MINSA, clinical follow-up must be carried out in all mild cases with or without risk factors during 14 days, the frequency is every 24 hours if it is remote and the face-to-face follow-up every 72 hours or earlier if the evolution Clinic requires it [12]. Most COVID-19 patients can be managed remotely with advice on symptomatic management and self-isolation; consultations can be made by phone, and the video also provides additional visual cues and therapeutic presence [20].

In a scenario where the response capacity of hospital centers has reached its limit, primary healthcare establishments considered as the gateway to the health system and that in Peru they represent $98 \%$ of all Establishments [21], which can take a more active role in controlling the pandemic. It is then necessary to strengthen the team that works in these establishments and improve the referralcounter-referral process to optimize limited resources, adding up the effort in the fight against the fearsome pandemic. Strong primary care systems form the basis of any emergency response, trained professionals can provide care across the spectrum of prevention, preparedness, response, and recovery; primary healthcare professionals must also participate in planning and action for risk management of health emergencies [22].

The following limitations should be considered in the study. The sample size has not allowed establishing relationships between the variables; due to the lack of uniformity in the complete filling in of medical records, the frequency of symptoms, treatment, vital functions, and home follow-up has been performed with less than the total number of participants. Despite these limitations, the study shows favorable results and can approximate the scenarios of primary healthcare establishments.

\section{Conclusions}

Evidence of an important response to COVID-19 is shown in the scenario of the first level of care. Mild to moderate cases can be managed on an outpatient basis and with home follow-up, contributing to the containment of the pandemic. Strengthen the first level of care to continue promoting preventive measures, diagnosis, treatment, and home follow-up, as well as improving the reference system for cases that require management in the hospital setting.

\section{Conflict of Interest}

The authors declare no conflict of interest.

\section{Acknowledgment}

Special thanks to Dr. Iván Vidal Puma and Glenys Olivos Lecarnaque from the "Mi Peru" Health Center, for the administrative advice.

\section{References}

[1] World Health Organization, Rolling updates on coronavirus disease (COVID-19), WHO, (Updated 25 May 2020), 2020.

[2] World Health Organization, Coronavirus Disease (COVID-19), 2020.

[3] Gobierno del Perú, Coronavirus en el Perú: casos confirmados, 2020.

[4] Ministerio de Salud, Sala Situacional COVID-19 Perú, Lima, Perú, 2020.

[5] L. Fu, B. Wang, T. Yuan, X. Chen, Y. Ao, T. Fitzpatrick, P. Li, Y. Zhou, Y. fan Lin, Q. Duan, G. Luo, S. Fan, Y. Lu, A. Feng, Y. Zhan, B. Liang, W. Cai, L. Zhang, X. Du, L. Li, Y. Shu, H. Zou, "Clinical characteristics of coronavirus disease 2019 (COVID-19) in China: A systematic review and meta-analysis," Journal of Infection, Apr 10, 2020, doi:10.1016/j.jinf.2020.03.041.

[6] B. Wang, R. Li, Z. Lu, Y. Huang, "Does comorbidity increase the risk of patients with covid-19: Evidence from meta-analysis," Aging, 12(7), 6049 6057, 2020, doi:10.18632/AGING.103000.

[7] A.J. Rodriguez-Morales, J.A. Cordona-Ospina, E. Gutiérrez-Ocampo, R. Villamizar-Peña, Y. Holguin-Rivera, "Clinical , laboratory and imaging features of COVID-19: A systematic review and meta-analysis," Travel Medicine and Infectious Disease, 34(March), 2020 , doi:10.1016/j.tmaid.2020.101623.

[8] Organización Internacional del Trabajo, El COVID-19 y el mundo del trabajo: repercusiones y respuestas, 2020.

[9] M. Nicola, Z. Alsafi, C. Sohrabi, A. Kerwan, A. Al-Jabir, C. Iosifidis, M. Agha, R. Agha, "The Socio-Economic Implications of the Coronavirus Pandemic (COVID-19): A Review," International Journal of Surgery, 78(March), 185-193, 2020, doi:10.1016/j.ijsu.2020.04.018.

[10] C. Scavone, S. Brusco, M. Bertini, L. Sportiello, C. Rafaniello, A. Zoccoli, L. Berrino, G. Racagni, F. Rossi, A. Capuano, "Current pharmacological treatments for COVID-19: what's next?," British Journal of Pharmacology, (April), 1-12, 2020, doi:10.1111/bph.15072.

[11] World Health Organization, Role of primary care in the COVID-19 response, 2020.

[12] Ministerio de Salud del Perú, Documento Técnico. Prevención y atención de personas afectadas por COVID-19 en el Perú, Perú, 2020.

[13] Asociación Médica Mundial, Declaración de Helsinki de la AMM-Principios éticos para las investigaciones médicas en seres humanos.

[14] S.P. Adhikari, S. Meng, Y. Wu, Y. Mao, R. Ye, Q. Wang, C. Sun, S. Sylvia, S. Rozelle, H. Raat, H. Zhou, "Epidemiology, causes, clinical manifestation and diagnosis, prevention and control of coronavirus disease (COVID-19) during the early outbreak period: a scoping review," Infectious Disease Poverty, 9(29), 1-12, 2020, doi:10.1186/s40249-020-00646-X.

[15] W.J. Guan, W.H. Liang, Y. Zhao, H.R. Liang, Z.S. Chen, Y.M. Li, X.Q. Liu, R.C. Chen, C.L. Tang, T. Wang, C.Q. Ou, L. Li, P.Y. Chen, L. Sang, W. Wang, J.F. Li, C.C. Li, L.M. Ou, B. Cheng, S. Xiong, Z.Y. Ni, J. Xiang, Y. Hu, L. Liu, H. Shan, C.L. Lei, Y.X. Peng, L. Wei, Y. Liu, et al., "Comorbidity and its impact on 1590 patients with COVID-19 in China: a 
nationwide analysis," The European Respiratory Journal, 55(March 2020), 2020, doi:10.1183/13993003.00547-2020.

[16] L. Panahi, M. Amiri, S. Pouy, "Risks of Novel Coronavirus Disease (COVID-19) in Pregnancy; a Narrative Review.," Archives of Academic Emergency Medicine, 8(1), e34, 2020.

[17] Y. Liu, H. Chen, K. Tang, Y. Guo, "Clinical manifestations and outcome of SARS-CoV-2 infection during pregnancy," Journal of Infection, (January 2020), 2020, doi:10.1016/j.jinf.2020.02.028.

[18] Ministerio de Salud del Perú, Modificación del documento de Prevención Diagnóstico y Tratamiento, RM 240-2020-MINSA, 2020.

[19] Ministerio de Salud del Perú, RM N 270-2020-MINSA, 2020.

[20] T. Greenhalgh, G.C.H. Koh, J. Car, "Covid-19: A remote assessment in primary care," The BMJ, 368(March 2020), 1-5, 2020, doi:10.1136/bmj.m1182.

[21] Ministerio de Salud, Plan Nacional de Fortalecimiento del Primer Nivel de Atención 2011 - 2021, Lima-Perú, 2011.

[22] C. Dunlop, A. Howe, D. Li, L.N. Allen, "The coronavirus outbreak: the central role of primary care in emergency preparedness and response," BJGP Open, 2020, doi:10.3399/bjgpopen20x101041. 